\title{
A license to cure?
}

\author{
Cathleen Lutz
}

\section{New genetic engineering technologies, as well as concerns from biotech and patient advocacy groups about access, are confronting the "business-as-usual" mentality of model sharing.}

\begin{abstract}
The past year has seen exciting developments in the rare disease community for patients with neuromuscular disorders. The FDA approved Sarepta's drug, Exondys 51, for use in treating Duchenne Muscular Dystrophy, marking a long awaited victory for this debilitating and deadly disease ${ }^{1}$. In Spinal Muscular Atrophy (SMA), clinical trials for therapies generated by two companies, Biogen and AveXis, demonstrated remark-
\end{abstract} able efficacy in Type I SMA infants. Babies that would have normally succumbed to the disease are remarkably achieving major developmental milestones and some, even walking 2 . Approval for Biogens's drug Spinraza came in the final weeks of 2016, with a broad level application in both pediatric and adult patients. Both Exondys 51 and Spinraza received orphan drug designation by the FDA.

These accomplishments are the result of many, many years of preclinical research using mouse models designed specifically to study these individual diseases. In SMA, dozens of mouse models were generated to under-

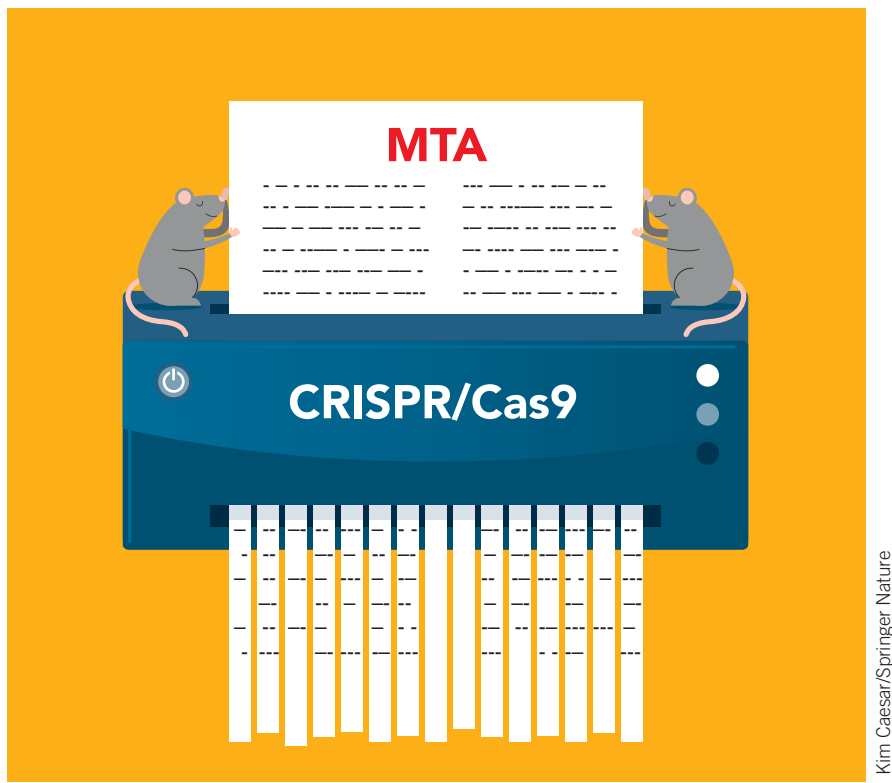

\section{Licensing mice}

The Bayh-Dole Act was enacted in 1980 to create a patent policy for universities, small businesses, and non-profits to retain the rights to inventions that were funded by the federal government. Many historians have heralded the Bayh-Dole Act as a catalyst for economic growth and the necessary incentive for academics to enter into financial relationships with industry, particularly in the pharmaceutical and biotechnology arenas. Notwithstanding these accomplishments, one of the major concerns surrounding the legislation was the access to research tools, which if too restricted, could greatly hamper downstream discoveries. As a result, the NIH issued guidelines to encourage the availability of research tools, recommending nonexclusive licensing and requiring grant recipients to develop resource sharing plans ${ }^{5}$. It was soon realized that broadly patenting mouse models was time consuming and generally not profitable. While many mouse models may not stand both the pathophysiology of the disease across the patient spectrum and for preclinical efficacy testing of potential therapeutics. Fortunately, the preclinical landscape is changing quickly and promising new technologies will shorten the time necessary to produce relevant preclinical models for both rare and common diseases. Whole genome sequencing in patients is dramatically increasing our knowledge of disease-causing mutations, revealing new molecular targets for therapy ${ }^{3}$. Advances in genome editing technologies, such as CRISPR/Cas9, allow scientists to generate preclinical mouse models faster, at less cost and containing these precise mutations found in the patients ${ }^{4}$. While these exciting developments promise significant hope to the patient community, a new bottleneck has emerged that threatens scientific and pharmaceutical progress-access to these newly developed mouse models for preclinical testing. meet the criteria for pursuing a patent, they are considered tangible research property and technology transfer offices (TTOs) usually require a material transfer agreement (MTA) and often, a license fee for use by for-profit companies ${ }^{6}$. This all seems perfectly fair and straight forward; the model creators control and reap the financial benefits of their research materials, while the for-profit sector will realize financial benefit should the model lead to a marketable therapeutic. However, the introduction of new gene editing technologies is rapidly changing the landscape of mouse models, not only in the pace and number by which we can generate the models, but the ability of the scientific community to adopt and validate these new models. With so many new models entering the pipeline, is the practice of negotiating individual agreements sustainable and is the barrier to entry for biotech companies too high, both in time and money? 


\section{The undeniable need}

One critical step for pharma and biotech companies in receiving approval from the FDA to advance a new therapy is establishing safety and efficacy in an animal model. With the number of mouse models available from public mouse repositories, one would think acquiring models for efficacy testing would be easy. Mice are indeed available to academics without restrictions. General terms and conditions are posted on repository websites, noting that the mice should be used for internal research purposes only and sharing of mice with a third party is prohibited. This is not the case for distribution to for-profit entities where the majority of donating institutions will require an agreement and fee for mice requested by industry.

This can be problematic for two reasons. First, the fees associated with these models can be costly, ranging anywhere from $\$ 20,000$ $\$ 100,000$ with additional annual charges ${ }^{6}$. For many start-ups, the cost is a definite consideration for working with emerging models, especially in the exploratory phase of the projects when the application of the model is still somewhat unknown. Second, and perhaps more important than dollars, is time. It can take months to negotiate licenses with TTOs with multiple "red-lining" of documents between parties before reaching an agreement. For models that possess more than one genetic variation, the time and expense can double if the genetic modifications are encumbered by different originating institutions.

Companies that have multiple diseases in their portfolio will logically move on one with which they can make the most progress. In working with companies that are exploring new therapeutic indications, one reoccurring question mouse repositories receive is: "What are the models that don't require a license?" Mouse models with arduous licenses not only serve as a show-stopper to some companies, but frustrate patients and foundations as well. Many patientbased foundations fund individual researchers to generate and characterize new models, only to learn later that their use by for profit companies will require a license from the originating institution. For many disease areas where the models are either not publically available or legally encumbered, the problem is significant enough to consider re-creating models using CRISPR/Cas9 technology and a third party contract research organization; many have already initiated this process. While some may argue that remaking mouse models is a waste of resources, the mere presence of the technology may put selective pressure on the community to reduce the current complexity in acquiring mouse models.

\section{An eye toward the future}

It is evident that discoveries within the last three years have afforded scientists the ability to generate models at a rate that would have seemed impossible even 10 years ago. The days of spending a couple of years on model development, followed by a few more years of publications with the model, and then finally resource sharing the model with a public repository and collecting license fees are dwindling. In this new era, the ease at which a mouse model can be recreated by others reduces its exclusivity if the model is not patented.

In the meantime, while we are flooding the mouse market with new models and negotiating license terms and fees, there are patients waiting; and for some, time is not on their side. What do we say to the mother with a new baby born with a rare mutation in ATP7A struggling with uncontrolled seizures; the 35 year old man recently diagnosed with ALS; the parents of a child born with an NGLY1 deficiency so rare there are only a handful of patients in the world? These are the people who may not have the time to wait while we constantly remake models or negotiate our way toward a curative therapy, and nor should they have to.

One solution is to seek out standardized electronic agreements; those that eliminate the negotiation process and greatly shorten the time and costs associated with putting the resource tools directly into the hands of those working toward therapies for disease. Reducing the fees associated with the licensing and/or providing industry with limited agreements to explore whether emerging mouse models fit their program is another avenue. TTOs will likely retain an even greater revenue stream by decreasing the risk to industry, which will result in increasing the volume of agreements. Emerging mouse models will then be more readily adopted and validated by the academic community, as well as industry, leading to both an improvement in reproducibility and translation of mouse data to the clinic. In doing so, licensing the cure will not come at the cost of time that could have been spent discovering the cure.

\section{COMPETING FINANCIAL INTERESTS}

The author declares no competing financial interests.

1. Syed, Y.Y. Eteplirsen: First global approval. Drugs 76, 1699-1704 (2016).

2. Adams, B. Early data win for AveXis' SMA Type 1 therapy but Biogen's med also strong. FierceBiotech. 2016; http://www.fiercebiotech.com/biotech/earlydata-win-for-avexis-sma-type-1-gene-therapy-but-biogen-figures-also-strong

3. Lohmann, K. \& Klein, C. Next generation sequencing and the future of genetic diagnosis. Neurotherapeutics 11, 699-707 (2014).

4. Low, B.E., Kutny, P.M. \& Wiles, M.V. Simple, Efficient CRISPR-Cas9-mediated gene editing in mice: Strategies and methods. Methods Mol. Biol. 1438, 19-53 (2016).

5. Ferguson, S. Licensing and distribution of research tools: National Institutes of Health perspective. J. Clin. Pharmacol. Suppl, 110S-112S (2001).

6. Dilling, M.B. \& Rakow, T.L. Licensing transgenic mice and other research tools: A practical guide. AUTM Technology Transfer Practice Manual, 3rd Edn. Vol 4, part 1 (2010). 